PAPER

\title{
The role of levodopa in the management of dementia with Lewy bodies
}

\author{
S Molloy, I G McKeith, J T O’Brien, D J Burn
}

J Neurol Neurosurg Psychiatry 2005;76:1200-1203. doi: 10.1136/jnnp.2004.052332

See end of article for authors' affiliations

.....................

Correspondence to: Dr D J Burn, Regional Neurosciences Centre, Newcastle General Hospital, Westgate Road, Newcastle upon Tyne NE4 6BE, UK; d.j.burn@ncl. ac.uk

Received 20 August 2004 In revised form

10 November 2004

Accepted

27 December 2004
Background: One of the core clinical features of dementia with Lewy bodies (DLB) is extrapyramidal syndrome (EPS). Levodopa is currently the gold standard oral therapy for Parkinson's disease (PD), but its use in DLB has been tempered by concerns of exacerbating neuropsychiatric symptoms.

Aim: To assess the efficacy and tolerability of L-dopa in managing EPS in DLB and to compare the motor response with that seen in PD and PD with dementia (PDD).

Method: EPS assessment consisted of the Unified Parkinson's Disease Rating Scale, motor subsection (UPDRS III), and finger tapping and walking tests. Patients with DLB were commenced on L-dopa. After 6 months, patients were examined in the "off" state, given L-dopa and assessed for motor responses. Identical assessments were performed in patients with PD and PDD also receiving L-dopa.

Results: Acute L-dopa challenge in 14 DLB patients yielded a mean 13.8\% ( $p=0.02)$ improvement in UPDRS III score, compared with $20.5 \%$ in PD $(n=28, p<0.0001)$ and $23 \%$ in PDD $(n=30, p<0.0001)$ respectively. Finger tapping scores increased (12.3\% v 20\% and 23\%), while walking test scores decreased (32\% v $41 \%$ and $67 \%$ ). Of the DLB patients, $36 \%$ were classified as "responders" on L-dopa challenge, compared with $70 \%$ of the PDD and $57 \%$ of the PD patients. Nineteen DLB patients were treated for 6 months with L-dopa (mean daily dose $323 \mathrm{mg}$ ). Two withdrew prematurely with gastrointestinal symptoms and two with worsening confusion.

Conclusion: L-dopa was generally well tolerated in DLB but produced a significant motor response in only about one third of patients. Younger DLB cases were more likely to respond to dopaminergic treatment.
$\mathrm{D}$ ementia with Lewy bodies is diagnosed according to consensus criteria when progressive cognitive decline is accompanied by two of three core clinical features (for probable DLB; one of three for possible DLB). These core features comprise well defined visual hallucinations, fluctuating cognition, and spontaneous parkinsonism. ${ }^{1}$ Up to $78 \%$ of DLB patients have extrapyramidal syndrome (EPS). ${ }^{2}$ Drugs used in the management of dementia, specifically cholinesterase inhibitors and neuroleptics, may worsen the EPS. ${ }^{3}$ Conversely, medications traditionally used to treat EPS can cause or worsen neuropsychiatric side effects, and thus have only been tentatively used in DLB to date for fear of precipitating or exacerbating an already fragile neurobehavioural state.

The most effective oral therapy available for Parkinson's disease is levodopa. Other dopaminergic therapies include dopamine agonists, anticholinergics, selegiline, and amantadine, but the side effects of these agents, including confusion, somnolence, and hallucinations, precludes their safe use in DLB. The benefit of L-dopa in the management of DLB is unclear, with most data available from retrospective, nonsystematic series. Studies have reported no, ${ }^{4}$ little, ${ }^{5}$ or marked improvement, ${ }^{6-8}$ while others concluded that the lack of a documented L-dopa response, or even a perceived need to treat with L-dopa, could be used as a distinguishing factor between PD and DLB. ${ }^{9}$ The aim of this study was therefore to ascertain prospectively whether DLB patients demonstrate Ldopa responsiveness and whether, over a 6 month therapeutic period, they were able to tolerate this medication. We compared L-dopa responsiveness in DLB patients with that elicited in PD patients with and without dementia.

\section{METHODS}

Patients

PD and DLB patients were recruited from hospital and community dwelling populations under the care of neurologists, old age psychiatrists, and geriatricians. The Newcastle and North Tyneside ethics committee approved the study, and all subjects gave informed consent to participate. Diagnosis was made by consensus between experienced clinicians using the consensus criteria for DLB ${ }^{1}$ and the UK Parkinson's Disease Society Brain Bank criteria for Parkinson's disease. ${ }^{10}$ Patients with PDD conformed to the Brain Bank criteria but also fulfilled the Diagnostic and statistical manual of mental disorders, 4th ed (DSM IV) criteria for dementia and clinical diagnostic criteria for probable DLB, developing more than 12 months after the onset of motor symptoms. ${ }^{1}$

The Mini Mental State Examination (MMSE) was used to provide a global measure of cognition. ${ }^{11}{ }^{12}$ Motor assessments comprised the Unified Parkinson's Disease Rating Scale part III (motor subsection), measurement of finger tapping times, and walking time tests, as recommended for monitoring Ldopa response. ${ }^{13}$ Patients already receiving L-dopa treatment were fasted overnight and assessed prior to their first daily dose of L-dopa, in a practically defined "off" state. ${ }^{14}$ Patients were then given co-beneldopa dispersible in a dose equivalent to their regular morning L-dopa dose (usually equivalent to one third of their total daily dose) and the acute motor effect was monitored every 30 minutes over the next 2 hours. Patients were then continued on their regular L-dopa dose and reassessed either on or off L-dopa, as tolerated, 6 months later.

If patients were not previously taking L-dopa this was commenced after their baseline motor assessment at a dose

Abbreviations: DLB, dementia with Lewy bodies; DSM IV, Diagnostic and statistical manual of mental disorders, 4th ed; EPS, extrapyramidal syndrome; MMSE, Mini Mental State Examination; PD, Parkinson's disease; PDD, Parkinson's disease with dementia; UPDRS, Unified Parkinson's disease Rating Scale 
of cocareldopa 12.5/50, three times daily and gradually titrated up according to dose response and side effect profile. These patients were fasted and assessed as described above in a practically defined off state 6 months after commencing treatment.

A positive L-dopa response was defined as a $>20 \%$ improvement in score in two of three tests of motor function, while a $<10 \%$ improvement was classified as L-dopa unresponsive. This was based upon the results of a metaanalysis of assessments of motor response in PD. ${ }^{15}$ An improvement of $<20 \%$ in UPDRS III score is also recognised as indicative of positive motor response to dopamine in drug naïve patients. ${ }^{16}$ The effects of L-dopa upon cognition, mood, and sleep in DLB will be the subject of another publication and are not discussed further here.

A single investigator ( $\mathrm{SM}$ ) performed all motor assessments, unblinded to diagnostic status. Inter-rater reliability for UPDRS scoring between the study rater and an experienced rater (DJB) was performed for 11 patients.

\section{Statistical analysis}

SPSS for Windows (version 11) was used for data analysis. All results were tested for normality using KolmogorovSmirnov test. For continuous variables, differences between groups were assessed using one way analysis of variance with post hoc Games-Howell analysis tests to determine group differences. For non-parametric data, Mann-Whitney $U$ test on two independent samples was used. All statistical tests were two tailed and were regarded as significant at $\mathrm{p}<0.05$. Inter-rater reliability for UPDRS ratings was assessed using a weighted kappa statistic that controls for chance agreement between raters, with a value of 1 indicating perfect agreement.

\section{RESULTS}

A total of 91 patients were included in this study, of whom 27 had DLB. Baseline demographic data for these patients is shown in table 1 , and compared with data for patients with PD $(n=31)$ and PDD $(n=33)$.

There was a male predominance in both the PD (24 men versus seven women) and PDD (23:10) groups, compared with the DLB group $(15: 12)\left(\chi^{2}=12.5, \mathrm{p}<0.001\right)$. The mean age of the PDD group (72.5 years) was less than either the PD (77.1 years, $\mathrm{p}<0.05)$ or DLB $(77.5$ years, $\mathrm{p}<0.05)$ groups, although, unsurprisingly, mean disease duration was longer in the PDD group ( 9.0 years compared with 4.9 years for PD and 2.7 years for DLB). MMSE scores in DLB and PDD groups were comparable $(18.5 \vee 20.2$, respectively, $\mathrm{p}=0.384)$ and both were less than the mean PD group score (26.0; $\mathrm{p}<0.0001$ ). Cholinesterase inhibitor use was $73 \%$ and $96 \%$, respectively, for the PDD and DLB groups.

Three PDD patients could not complete a fasting motor assessment due to severe motor disability, and three patients with PD did not require L-dopa. Baseline motor data for the remaining patients is presented in table 2. This combines patients assessed in a practically defined "off" state with those who required but who had not yet commenced L-dopa. Given the longer disease duration and the fact that only six of the 27 DLB patients were taking L-dopa at baseline, it is not surprising that the mean L-dopa dose was significantly greater in the PDD group $(\mathrm{p}=0.001 ; 590 \mathrm{mg}$ per day, compared with $98 \mathrm{mg}$ for DLB and $257 \mathrm{mg}$ for PD groups).

Inspection of table 2 also reveals that the PDD group had the most significant motor impairment in all parameters assessed. There was no significant intergroup difference in the walking time step second product, although the large standard deviations made these data difficult to interpret. The greater product in the PDD group could be interpreted as reflecting their greater disease duration, however. The evidence for more severe EPS in PDD persisted using the UPDRS- 5 subscale (calculated using the sum of total scores from five items of the full UPDRS III, previously shown to be independent of the severity of cognitive impairment). ${ }^{17}$ Interrater reliability testing confirmed a high level of agreement between assessors for 11 cases checked for UPDRS III (average measure of intraclass correlation of 0.9775; confidence interval 95\%).

\section{Acute L-dopa challenge}

Acute L-dopa challenges were performed in 14 of the 27 DLB patients. Reasons for the other 13 DLB patients not receiving this challenge were: patient previously on L-dopa but medically incapable of completing repeated assessments over a 3 hour study period $(\mathrm{n}=4)$; patient receiving L-dopa de novo but declined or was medically incapable of completing an overnight fast and acute, repeated motor assessments $(\mathrm{n}=3)$; patient receiving L-dopa de novo but not tolerated, therefore acute fasting challenge not performed $(n=4)$; referring physician requested L-dopa not be administered $(\mathrm{n}=1)$; and severe symptomatic orthostatic hypotension precluding introduction of L-dopa $(\mathrm{n}=1)$. Of 14 DLB patients assessed acutely, two had previously received L-dopa prior to study entry, while 12 were commenced on L-dopa as part of this study. The mean dose of L-dopa administered for the challenge to the DLB group was $103 \mathrm{mg}$, and this did not differ significantly from the dose administered to the PDD (115 mg) and PD (101 $\mathrm{mg}$ ) patients.

The magnitude of motor response to L-dopa was reduced in the DLB group for all parameters tested, compared with the PD and PDD patients (table 3). These differences reached statistical significance for the UPDRS III $(p<0.05)$ and finger tapping $(\mathrm{p}<0.05)$, with the improvement in DLB significantly less in both tests compared with that seen in PDD $(p<0.05)$.

Although an improvement of $>20 \%$ was seen in the walking time test for the DLB group, this is of uncertain significance due to the wide standard deviation. The UPDRS III, UPDRS-5, and finger tapping responses did not differ significantly between the PD and PDD groups, although all improvements were modest, presumably reflecting the relatively conservative L-dopa dose used for the challenges.

The L-dopa response of each patient was assessed, with "response" defined as a greater than $20 \%$ improvement over baseline on two of the three motor assessments (as detailed above). When the data were analysed in this way, only $36 \%$ of the DLB group was classified as L-dopa responsive, compared with $70 \%$ of the PDD group and $57 \%$ of the PD 
Table 2 Baseline motor data for DLB, PDD, and PD patient groups

\begin{tabular}{|c|c|c|c|c|c|c|}
\hline Group (n) & $\begin{array}{l}\text { L-dopa } \\
\text { naive (n) }\end{array}$ & $\begin{array}{l}\text { Daily L- } \\
\text { dopa (mg) }\end{array}$ & UPDRS III & UPDRS-5 & FT & WT (steps/s) \\
\hline $\mathrm{DLB}(27)$ & 21 & $98(188) \dagger$ & $35.7(12.3)$ & $14.3(6.4)$ & $23.5(14.4)$ & $761.9(1978)$ \\
\hline PDD (30) & 0 & $590(414)$ & $42.9(13.4) \dagger$ & $18.7(7.5)^{*}$ & 26.5 (10.5) & $1620(3300)$ \\
\hline PD (28) & 9 & $257(241)$ & $32.7(9.2)$ & $15.7(5.6)$ & $40.65(9.4) \ddagger$ & 801 (1717) \\
\hline
\end{tabular}

group (table 4). Conversely, $29 \%$ of the DLB were classified as unresponsive, compared with $10 \%$ PDD and $21 \%$ PD patients (table 4).

Comparison between L-dopa responding and non-responding DLB patients revealed no difference in sex, disease duration, MMSE, UPDRS, five item UPDRS, or dose of L-dopa achieved. L-dopa responders were, however, younger (68.5 v 77.1 years, $\mathrm{p}=0.014)$.

\section{Chronic L-dopa use (tolerability)}

Of the 27 patients with DLB, one did not commence L-dopa at the request of the physician owing to fear of neurobehavioural sequelae (UPDRS $=38$ ) and one had severe symptomatic orthostatic hypotension. Thus, 25 of the 27 DLB patients were chronically treated with L-dopa. Six of these were already taking this medication at study entry. Of the 25 DLB patients, $82 \%(\mathrm{n}=21)$ warranted and tolerated long term L-dopa therapy. Nineteen patients with DLB were therefore commenced de novo on L-dopa for the purposes of this study, with a view to chronic dosing over at least 6 months. The six patients already taking L-dopa did not differ significantly in age, disease duration, MMSE, or baseline UPDRS from those not taking L-dopa at baseline assessment (data not shown).

Of the 19 patients, two were unable to tolerate L-dopa within the first 2 weeks due to worsening confusion $(n=1)$ and severe exacerbation of hallucinations $(\mathrm{n}=1)$. A further two patients withdrew before the 3 month assessment, owing to gastrointestinal intolerance, nausea, abdominal pain, and bloating. All side effects resolved once the dopaminergic medication was stopped. Fifteen DLB patients $(84 \%)$ were therefore able to tolerate L-dopa for the 6 month study duration. The mean daily L-dopa dose achieved was 323 (182) mg. None of the DLB patients either previously receiving or those commenced de novo on L-dopa developed dyskinesias during the study period.

\section{DISCUSSION}

The main conclusion from this study is that L-dopa, while generally well tolerated in modest doses by DLB patients, is not particularly efficacious in alleviating the parkinsonian syndrome seen in this disorder. Younger DLB patients may be more likely to experience a beneficial response. Thus, while $82 \%$ of 25 DLB patients were able to remain on a mean daily dose of $323 \mathrm{mg}$ L-dopa for 6 months, formal L-dopa challenges indicated that only $36 \%$ of 14 patients tested could be classified as "responders", according to previously published criteria. ${ }^{15}$ This contrasts with the 56 and 70\% response rates in PD and PDD, respectively. Others have recently reported similar

Table 3 Group changes in motor parameters with acute L-dopa challenge

\begin{tabular}{llllll}
\hline Group (n) & $\begin{array}{llll}\text { L-dopa } \\
\text { (mg (SD)) }\end{array}$ & $\begin{array}{l}\% \Delta \\
\text { UPDRS }\end{array}$ & $\begin{array}{l}\% \Delta \\
\mathbf{5} \text { item }\end{array}$ & $\begin{array}{l}\% \Delta \\
\text { FT }\end{array}$ & $\begin{array}{l}\% \Delta \\
\text { WT }\end{array}$ \\
\hline DLB (14) & $103.3(51.6)$ & $13.8^{*}$ & 23 & $12.3^{*}$ & 31.5 \\
PDD (30) & $115(45.8)$ & 23 & 37 & 23 & 67 \\
PD (28) & $101.8(21.4)$ & 20.5 & 34 & 19.5 & 41.4 \\
\hline
\end{tabular}

findings to the present study, with reduced motor improvement on formal levodopa testing in DLB patients. ${ }^{18}$

Variable dopaminergic responsiveness has been reported in observational studies of DLB, although the retrospective nature, lack of control groups and non-standardised assessment of motor function make these data difficult to interpret. ${ }^{4-9}$ The lack of a documented L-dopa response, or a perceived need to treat with L-dopa has been suggested to be of use as a distinguishing factor between PD and DLB. ${ }^{9}$ In contrast, all 12 DLB patients in another study were deemed to be L-dopa responsive, although this was not quantified. ${ }^{4}$ More recently, L-dopa therapy was initiated in $48 \%$ of 40 DLB patients at a mean dose of $525 \mathrm{mg} /$ day, with a "good" motor response noted in $66 \%{ }^{8}$ Efficacy was maintained for a mean follow up of 3.2 years, although dyskinesias developed in $16 \%$ of the patients. This study was, however, a retrospective design and motor response was again not quantified.

There are several reasons why DLB patients might be expected to respond less well to dopaminergic treatments, including intrinsic striatal $\alpha$-synuclein pathology ${ }^{19}$ and reduced caudal putamen dopamine D2 receptors. ${ }^{20}$ More recently, loss of dopamine D3 receptors on corticostriate projection neurones has also been proposed as a possible mechanism for L-dopa refractoriness. ${ }^{21}$ Alternatively, the emergence of so-called "non-dopaminergic" motor features, mediated by degeneration in other brainstem nuclei such as the pedunculopontine nucleus, would be predicted to give a reduced response to L-dopa therapy.

Interestingly, a similar loss of dopaminergic responsiveness has also been suggested to occur in $\mathrm{PDD}_{,}^{22}{ }^{23}$ and clinical data suggest that non-dopaminergic motor features may, indeed, be over-represented in both PDD and DLB. ${ }^{24}$ In the present study, however, the PDD group showed a 70\% overall response to the acute L-dopa challenge, with only $10 \%$ of the 30 patients tested being classified as non-responders. Although UPDRS III scores were highest in this group, compared with the PD and DLB patients, the mean disease duration was still relatively short at 9.0 years. The contrast in L-dopa responsiveness between DLB and PDD patients in this study highlights a potentially important clinical difference between these conditions. ${ }^{25}$ Further work is required to determine the pathophysiological mechanisms underpinning these observations.

A responder frequency of only $57 \%$ in the PD group, with $21 \%$ classified as non-responders, is somewhat surprising. This apparent poor response rate in PD may reflect the low

Table 4 Responder and non-responder frequency with acute L-dopa challenge

\begin{tabular}{llllll}
\hline Group (n) & $\begin{array}{l}\text { UPDRS } \\
\text { III }>\mathbf{2 0} \%\end{array}$ & $\begin{array}{l}\text { FT } \\
>\mathbf{2 0} \%\end{array}$ & $\begin{array}{l}\text { WT } \\
>\mathbf{2 0} \%\end{array}$ & Responders & $\begin{array}{l}\text { Non- } \\
\text { responders }\end{array}$ \\
\hline DLB (14) & 5 & 4 & 9 & $5(36 \%)$ & $4(29 \%)$ \\
PDD (30) & 16 & 20 & 21 & $21(70 \%)$ & $3(10 \%)$ \\
PD (28) & 14 & 14 & 14 & $16(57 \%)$ & $6(21 \%)$ \\
\hline
\end{tabular}

Responder: defined as a group of patient manifesting $>20 \%$ improvement in two or more of the three motor tests after introduction of L-dopa therapy. Non-responder: defined as $<10 \%$ improvement in two or more of the three motor tests. FT, finger tapping; WT, walking test. 
dose titration employed in the one third of the PD group that were introduced to L-dopa de novo. This, in turn, led to the administration of a lower than normal L-dopa dose for the acute challenge (mean $101 \mathrm{mg}$ ), whereas most other studies have advocated a supranormal dose challenge (of at least $250 \mathrm{mg}) .{ }^{26}$ The reason for this choice was to make the acute response between DLB and the other groups as comparable as possible. It was felt that using higher L-dopa doses in frail and cognitively impaired patients would run an unacceptably high risk of exacerbating or causing neuropsychiatric complications. A further potential explanation is the fact that the PD patients performed particularly well on the finger tapping test at baseline, and thus there may have been a "ceiling effect" in response to L-dopa on this measure.

There are several drawbacks to this study. It was an open label design, therefore any results are subject to a degree of bias, while some of the benefits of L-dopa use may reflect a placebo response. Many of the patients recruited were elderly and frail. Physical and cognitive disability was profound in some and this may have rendered the interpretation of tests results unreliable. Test conditions were not always standardised, and while the finger tapping test and UPDRS are less subject to environmental influence, assessments were often carried out in the patient's home, making the 6 metre walk test prone to increased variability. Furthermore, only 14 of 27 (52\%) DLB patients were able to undergo an acute L-dopa challenge, potentially limiting the generalisability of our results. The doses of L-dopa used throughout this study were relatively conservative and we could not exclude a greater motor response with higher doses. It is noteworthy that all but one of the DLB patients were taking cholinesterase inhibitors when exposed to L-dopa, so we are unable to comment upon the tolerability of dopaminergic treatment in DLB patients not taking cholinesterase inhibitors. Although we found that younger DLB patients may respond better to Ldopa, the relatively small numbers do not permit a firm conclusion to be made in this regard.

Despite these drawbacks, the study has a number of advantages. To the best of our knowledge, this is the first time that DLB patients have been prospectively assessed both acutely and chronically for L-dopa responsiveness and tolerability. A single assessor performed all the motor tests, thereby eliminating interobserver variability. The inclusion criteria were broad, so the study population recruited and conclusions drawn are likely to represent the "real life" situation faced by clinicians in their day to day practice.

In conclusion, we suggest that L-dopa can be employed in the management of EPS in DLB without producing significant or irreversible side effects, improving parkinsonism in approximately one third. Younger DLB patients may benefit most from this treatment. Furthermore, this study has produced evidence to support the continued use of Ldopa in patients with PDD, as motor benefits were still apparent despite the superadded cognitive decline.

\section{ACKNOWLEDGEMENTS}

Our thanks to E N Rowan for her valued statistical review and to A Nicholson and L Bowman for help with database formation.

\section{Authors' affiliations \\ S Molloy, I G McKeith, J T O’Brien, D J Burn, University of Newcastle upon Tyne, Newcastle, UK}

Competing interests: none declared

\section{REFERENCES}

1 McKeith IG, Galasko D, Kosaka K, et al. Consensus guidelines for the clinical and pathologic diagnosis of dementia with Lewy bodies (DLB): Report on the consortium on DLB international workshop. Neurology 1996:47:1113-24.

2 McKeith IG. Dementia with Lewy bodies: Clinical and pathological diagnosis. Alzheimer Rep 1998;1:83-7.

3 Richard IH, Justus AW, Greig NH, et al. Worsening of motor function and mood in a patient with Parkinson's disease after pharmacologic challenge with oral rivastigmine. Clin Neuropharmacol 2002;25:296-9.

4 Gnanalingham KK, Byrne EJ, Thornton A, et al. Motor and cognitive function in Lewy body dementia: comparison with Alzheimer's and Parkinson's diseases. J Neurol Neurosurg Psychiatry 1997;62:243-52.

5 Gibb WRG, Luthert PJ, Janota I, et al. Cortical Lewy body dementia: clinical features and classification. I Neurol Neurosurg Psychiatry 1989;52:185-92.

6 Byrne EJ, Lennox G, Lowe J, et al. Diffuse Lewy body disease: clinical features in 15 cases. J Neurol Neurosurg Psychiatry 1989;52:709-17.

7 Louis ED, Goldman JE, Powers JM, et al. Parkinsonian features of eight pathologically diagnosed cases of diffuse Lewy body disease. Mov Disord 1995; 10:188-94.

8 Rojo AA, Aguilar MM, Navas II, et al. Parkinsonism and the response to treatment in dementia with Lewy bodies. Mov Disord 2002; 17(suppl 5):S257-8.

9 Louis ED, Klatka LA, Liu Y, et al. Comparison of extrapyramidal features in 31 pathologically confirmed cases of diffuse Lewy body disease and 34 pathologically confirmed cases of Parkinson's disease. Neurology 1997;48:376-80.

10 Gibb WRG, Lees AJ. The relevance of the Lewy body to the pathogenesis of idiopathic Parkinson's disease. J Neurol Neurosurg Psychiatry 1988;51:745-52

11 Folstein M, Folstein S, McHugh PR. "Mini-mental state". A practical method for grading the cognitive state of patients for the clinician. $J$ Psychiatr Res 1975; 12:189-98.

12 Clarke M, Jagger C, Anderson J, et al. The prevalence of dementia in a total population: a comparison of two screening instruments. Age Ageing 1991;20:396-403.

13 Defer GL, Widner H, Marie RM, et al. Core assessment program for surgical interventional therapies in Parkinson's disease (CAPSIT-PD). Mov Disord 1999;14:572-84.

14 Langston JW, Widner H, Goetz CG, et al. Core assessment program for intracerebral transplantations (CAPIT). Mov Disord 1992;7:2-13.

15 Clarke C, Davies P. Systematic review of acute levodopa and apomorphine challenge tests in the diagnosis of idiopathic Parkinson's disease. J Neurol Neurosurg Psychiatry 2000;69:590-94.

16 Gasser T, Schwarz J, Arnold G, et al. Apomorphine test for dopaminergic responsiveness in patients with previously untreated Parkinson's disease. Arch Neurol 1992;49:1131-4.

17 Ballard C, McKeith I, Burn D, et al. The UPDRS scale as a means of identifying extrapyramidal signs in patients suffering from dementia with Lewy bodies. Acta Neurol Scand 1997;96:366-71.

18 Bonelli SB, Ransmayr G, Steffelbaver M. L-dopa responsiveness in dementia with Lewy bodies, Parkinson's disease with and without dementia. Neurology 2004;63:376-8.

19 Duda JE, Benoit BI, Meghann EM, et al. Novel antibodies to synuclein show abundant striatal pathology in Lewy body diseases. Ann Neurol 2002;52:205-10.

20 Piggott MA, Marshall EF, Thomas N, et al. Striatal dopaminergic markers in dementia with Lewy bodies, Alzheimer's and Parkinson's diseases: rostrocaudal distribution. Brain 1999;122:1449-68.

21 Joyce JN, Ryoo HL, Beach TB, et al. Loss of response to levodopa in Parkinson's disease and co-occurrence with dementia: role of D3 and not D2 receptors. Brain Res 2002;95:138-52.

22 Growdon JH, Kieburtz K, McDermott P, et al. Levodopa improves motor function without impairing cognition in mild non- demented Parkinson's disease patients. Neurology 1998;50:1327-31.

23 Apaydin H, Ahlskog JE, Parisi JE, et al. Parkinson disease neuropathology: later developing dementia and loss of levodopa response. Arch Neurol 2002;59:102-12.

24 Burn DJ, Rowan EN, Minett T, et al. Extrapyamidal features in Parkinson's disease with and without dementia and dementia with Lewy bodies: a cross sectional comparitive study. Mov Disord 2003;18:884-9.

25 Aarsland D, Ballard CG, Halliday G. Are Parkinson's disease with dementia and dementia with Lewy bodies the same entity? J Geriatr Psychiatry Neurol 2004; 17:137-45

26 Merello M, Nouzeilles MI, Piran Arce G, et al. Accuracy of acute levodopa challenge for clinical prediction of sustained long-term levodopa response as a major criterion for idiopathic Parkinson's disease diagnosis. Mov Disord 2002;17:795-8. 